\title{
Interação síncrona na Educação a Distância a partir do olhar dos estudantes
}

\author{
Students evaluation of synchronous interactions in \\ distance education
}

\author{
IGOR THIAGO MARQUES MENDONÇA \\ Instituto Federal de Santa Catarina (IFSC)
}

CRISLAINE GRUBER Instituto Federal de Santa Catarina (IFSC)

\begin{abstract}
Resumo: Os momentos de interação síncrona entre professores e estudantes oportunizam discussões, troca de experiências e colaboração na educação a distância. Considerando a importância desses momentos e a multiplicidade de fatores que os influenciam, este trabalho avalia a percepção dos estudantes da EAD em relação às interações síncronas realizadas com auxílio de teleconferências. A partir de questionário, foram coletadas opiniões de 163 estudantes, 100 que utilizam Webconferência e 63 que utilizam Videoconferência. Foi utilizada a estatística descritiva para elaborar uma análise comparativa entre as duas tecnologias. As interações feitas por Webconferência foram melhor avaliadas nos aspectos qualidade e quantidade de interações, adequação do ambiente e contribuição para formação profissional. Os estudantes indicaram a necessidade de melhorias na transmissão das Videoconferências. A influência do professor é alta independente da tecnologia utilizada. Observou-se um importante engajamento dos estudantes na pesquisa, os quais sugeriram melhorias para as interações síncronas.
\end{abstract}

Palavras-chave: Educação profissional. Ensino a distância. Educação online. Recomendações para EAD. Tecnologias educacionais.

Abstract: Synchronous interactions between teachers and students are opportunities for discussion, exchange of experiences and collaboration in distance education. Based on the importance of these moments and the multiplicity of factors that influence them, this study evaluates students perception of the synchronous interactions in distance education carried out by teleconferences. We collected the opinions of 163 students, 100 who use Webconference and 63 who use Videoconference. We used descriptive statistics to analyze the data and we made comparisons between the two technologies. The interactions made by Webconference were better evaluated in terms of quality and quantity of interactions, suitability of the environment and contribution to professional training. The students indicated the need for improvements in the transmission of Videoconferences. Teachers influence is high regardless of the technology used. We observed a significant engagement of the students in the research; they made suggestions of improvements for synchronous interactions.

Keywords: Professional education. Distance education. Online education. Recommendations for distance education. Educational technologies.

MENDONÇA, Igor Thiago Marques; GRUBER, Crislaine. Interação síncrona na Educação a Distância a partir do olhar dos estudantes: uma análise comparativa entre Webconferência e Videoconferência. Informática na Educação: teoria \& prática, Porto Alegre, v. 22, n. 2, p. 159-174, maio./ago. 2019. 


\section{Introdução}

A Educação a Distância (EAD) no Brasil manteve o ritmo de crescimento nos últimos anos. Segundo dados do censo da educação superior, o número de ingressantes em cursos desta modalidade foi de quase 1,4 milhões em 2015 (INEP, 2018), contabilizando 17,4\% de todos os cursos de ensino superior no país. A Associação Brasileira de Educação a Distância divulgou que as matrículas em cursos de EAD em 2017, para os diversos níveis de ensino, superaram 7,7 milhões (ABED, 2018). A EAD pode ser ofertada totalmente a distância ou no modelo semipresencial. Outra característica que diferencia os cursos de EAD são as modalidades de mediação pedagógica com ou sem tutores, nas quais os professores contam, ou não, com uma equipe de pessoas para auxiliá-los no decorrer do curso.

A Universidade Aberta do Brasil (UAB), uma iniciativa do governo brasileiro para incentivar a adoção da EAD pelas universidades públicas, adotou a modalidade de mediação pedagógica com auxílio de tutores presenciais (um a cada 25 estudantes, atuando nos polos de apoio presencial) e tutores a distância (atuando junto aos professores na proporção de um para cada 25-35 estudantes), o que permite o atendimento a um grande número de estudantes (CASTRO; CARULLA; MELO, 2014). A interação síncrona entre estudantes e professores nos cursos da UAB é feita semanalmente por meio de Videoconferências (VideoC) transmitidas nos polos de apoio presencial.

O Instituto Federal de Santa Catarina (Ifsc) oferta cursos por meio do programa UAB. Contudo, iniciou paralelamente em 2014 um processo de institucionalização da EAD. Para isso, criou um Centro de Referência em Formação e EAD (Cerfead), com quadro próprio de servidores, vinculado à Pró-Reitoria de Ensino. O Cerfead, que tem dentre seus objetivos contribuir com a ampliação e consolidação da oferta formativa dos campi do Ifsc por meio da EAD, está em fase de implantação e de experimentação de alguns modelos de EAD (Ifsc, 2018). As principais diferenças dos cursos do Ifsc em relação ao modelo UAB é a ausência de tutores, sendo a mediação realizada pelos próprios professores, e a principal ferramenta de interação síncrona é a Webconferência (WebC), extinguindo a necessidade de os estudantes se locomoverem a polos de apoio presencial semanalmente. Para viabilizar a mediação realizada exclusivamente pelos professores, utiliza-se no Cerfead a estratégia de organizar as turmas em grupos de 40 a 60 estudantes, atribuindo ao professor uma carga horária equivalente àquela que ele teria em cursos presenciais para cada um desses grupos. Por exemplo, em um curso com turmas de 160 estudantes, considera-se que o professor está trabalhando com quatro grupos de 40 estudantes, permitindo assim que ele tenha carga horária para desenvolver seu trabalho com qualidade, dando feedback, interagindo, corrigindo atividades etc. Haas e Lopes (2014), em pesquisa realizada junto a professores da EAD, constataram que uma quantidade elevada de estudantes por turma prejudica a qualidade do ensino, dificultando a atenção e a correção de muitas atividades. 
Independente do modelo de mediação, a interação com os estudantes na EAD ocorre de forma síncrona ou assíncrona. As ferramentas de interação assíncrona são desconectadas de tempo e espaço, ou seja, o estudante interage no seu tempo e ritmo. Exemplos delas são fóruns, e-mails, vídeo-aulas, textos, blogs etc. As ferramentas síncronas ocorrem em tempo real e exemplos delas são os chats ou teleconferências (audioconferências, VideoC e WebC). Seu uso pressupõe uma estratégia definida no projeto pedagógico do curso. Por ser um momento síncrono do professor com os estudantes, espera-se que ocorra algum tipo de interação; caso contrário essa ferramenta poderia ser substituída por um vídeo gravado. As interações têm impacto importante na efetividade de cursos EAD (BOTELHO; VICARI, 2009). Depende dessa interação a qualidade da presença dos estudantes e professores no ambiente virtual de aprendizagem (LUNKES; SELLI; PRATES, 2008).

As características intrínsecas das ferramentas utilizadas para realização das interações síncronas podem colaborar ou não para que as interações ocorram. Por exemplo, para participar das WebC o estudante encontra-se num espaço que ele mesmo escolheu, enquanto que para participar das VideoC o espaço é o polo de apoio presencial. Estar sozinho em frente ao seu computador pode ser uma vantagem para os estudantes mais tímidos. Além disso, evitar o trânsito ou transporte para chegar ao local de aula pode ser uma forma de redução do estresse. Em contraposição, acessar e configurar uma WebC pode ser um problema para as pessoas com menor experiência em tecnologias da informação e comunicação (TICs).

Considerando a importância dos momentos de interação síncrona na EAD e a complexidade de fatores que podem influenciá-los, esta pesquisa objetiva avaliar a percepção dos estudantes de cursos EAD em relação às interações síncronas realizadas com auxílio de teleconferências. $O$ processo de institucionalização da EAD no Ifsc instigou os autores a conduzir a pesquisa tanto com estudantes que utilizam a VideoC quanto com os que utilizam a WebC, comparando as duas tecnologias a fim de alimentar as discussões sobre esse assunto e aprimorar as interações síncronas.

\section{Materiais e métodos}

Optou-se pelo instrumento de pesquisa inquirição, por meio de questionário, para avaliar a percepção dos estudantes em relação aos diversos aspectos de uso e interação das teleconferências. O questionário permitiu também traçar os perfis dos participantes com questões sobre sexo, idade, nível de instrução, atividade profissional, atuação na área de educação, experiência com TICs e quantidade de cursos EAD já realizados. A partir desse perfil, foi avaliado se as características do participante têm influência em sua percepção de uso das teleconferências. Os professores da área de Tecnologias para Educação do Cerfead avaliaram as questões e suas sugestões foram incorporadas. Na sequência, houve uma primeira rodada de respostas com estudantes do curso de Especialização em Tecnologias para Educação Profissional, seguida de pequenos ajustes para que o questionário fosse disponibilizado para os demais cursos. 
O questionário online ficou disponível para participação voluntária e anônima dos estudantes nos ambientes virtuais de aprendizagem dos cursos no período de $6 / 8$ a $18 / 10$ de 2018 . Foram selecionadas as especializações de oferta própria do Cerfead, Docência para a Educação Profissional (WD), Gestão Pública para a Educação Profissional e Tecnológica (WG) e Tecnologias para Educação Profissional (WT), que usam WebC, totalizando 100 participantes, e três cursos da UAB, Especializações em Formação pedagógica para EPT (VF) e em Gestão Pública (VG) e Licenciatura em EPT (VL), que usam VideoC, totalizando 63 participantes. As siglas que acompanham os nomes dos cursos foram criadas pelos autores para facilitar a identificação no artigo.

A maior parte das questões sobre a percepção dos participantes foi elaborada usando a escala likert de 1 a 4 ou 1 a 5 em que a nota 1 corresponde a uma baixa influência ou satisfação e a nota 5 corresponde a uma alta influência ou satisfação. A Tabela 1 apresenta as questões e seus objetivos. Ao final do questionário, foi disponibilizado um campo de texto para que os estudantes pudessem fazer comentários sobre sua experiência com as teleconferências.

Tabela 1 - Questões que avaliaram a percepção dos estudantes frente à tecnologia de teleconferência usada e objetivos de cada questão.

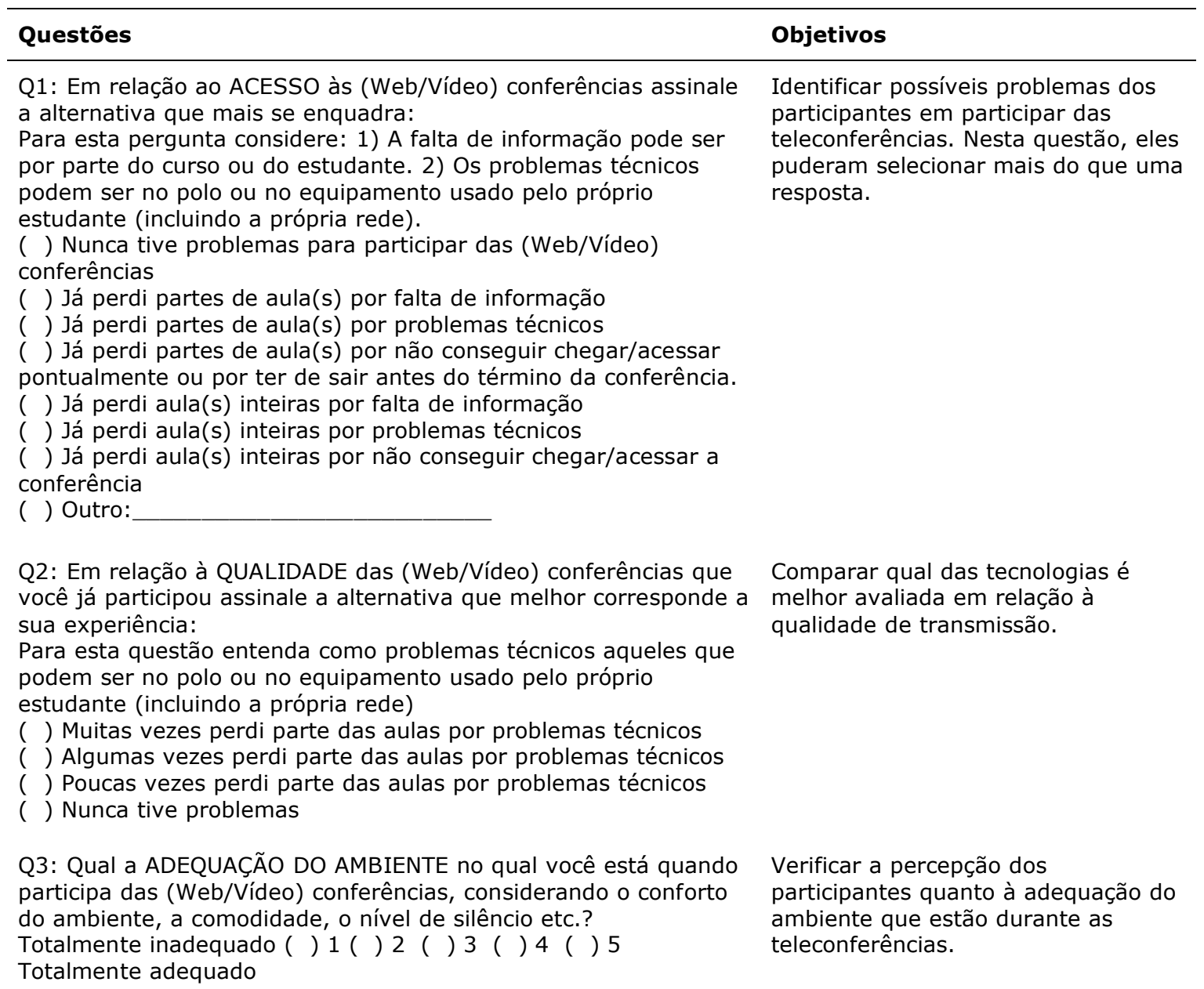


Q4: Na sua opinião, em que medida o(s) professor(es) influencia $(m)$ na qualidade das interações síncronas por meio de (Web/Vídeo) conferência?

Não influenciam ( ) 1 ( ) 2 ( ) 3 ( ) 4 ( ) 5 Influenciam totalmente

Q5: A (Web/Vídeo) conferência é uma ferramenta que pressupõe interação entre o professor e os estudantes. Diante disso, como você classifica a QUANTIDADE DE INTERAÇÕES das (Web/Video) conferências que participou?

Totalmente inadequada ( ) 1 ( ) 2 ( ) 3 ( ) 4 ( ) 5

Totalmente adequada

Q6: Qual o seu grau de SATISFAÇÃO quanto aos meios usados pelos professores para interagir com os estudantes nas (Web/Vídeo) conferências que participou?

Totalmente insatisfeito ( ) 1 ( ) 2 ( ) 3 ( ) 4 ( ) 5 Totalmente satisfeito

Q7: Qual o nível de contribuição das (Web/Vídeo) conferências na formação profissional que você está realizando ou realizou? Irrelevante ( ) $1($ ) $2($ ) $3($ ) 4 ( ) 5 Muito relevante
Identificar a percepção dos participantes quanto à qualidade da teleconferência depender do professor.

Identificar se os participantes estão satisfeitos com a quantidade de interações realizadas com o recurso de teleconferência.

Verificar o grau de satisfação quanto ao método de uso da teleconferência, pois a forma como ocorrem as interações pode influenciar na percepção do estudante quanto à qualidade das aulas.

Avaliar a percepção dos participantes quanto à contribuição da teleconferência para sua formação profissional, visto que o público atendido pelo Cerfead é predominantemente de trabalhadores.

Fonte: os autores.

As respostas coletadas foram inseridas em uma planilha eletrônica para proceder à realização dos cálculos e gráficos. Para a análise dos dados foram usados indicadores da estatística descritiva como média, desvio padrão, moda, coeficiente de variação e coeficiente de correlação de Pearson. Além disso, criou-se um índice para cada participante, o qual permitiu traçar um panorama da sua percepção por tecnologia utilizada. Esse índice equivale à média das notas atribuídas às questões sobre adequação do ambiente (Q3), quantidade de interações (Q5), satisfação (Q6) e contribuição para sua formação profissional (Q7). O coeficiente de correlação de Pearson foi usado para testar a correlação entre algumas das respostas, podendo ser baixa, média ou alta.

\section{Resultados e discussões}

Dentre os 163 participantes do estudo, 59\% são do sexo feminino e 41\% masculino; 76\% têm entre 26 e 45 anos, 15\% entre 46 e 55 e 9\% têm mais de 55 ou menos de 26 anos. Todos os participantes têm nível de escolaridade superior, sendo que 55\% possuem pós-graduação. Em relação à atividade profissional, 64\% declararam trabalhar na iniciativa pública, 27\% na iniciativa privada, 6\% são autônomos e 3\% estão sem atividade profissional. Quase metade da amostra (48\%) já atuou mais de 3 anos na área de educação; 21\% atuaram menos de 3 anos nesta área e 31\% nunca atuou. Quanto ao tempo conectados à internet diariamente, 77\% dos participantes declararam estar conectados o tempo todo, $19 \%$ de 2 a 4 horas e somente $4 \%$ de 
1 a 2 horas. A maior parte dos participantes demonstrou conhecimento a respeito de ferramentas e serviços da internet como e-mail, navegadores, chats, VideoC e redes sociais.

Com base nos dados levantados sobre o perfil dos participantes, pode-se concluir que profissionais ativos (97\% da amostra), que possuem graduação ou pós-graduação, continuam em busca de aperfeiçoamento profissional e, para isso, encontram como uma alternativa a EAD. No que tange à atividade profissional, percebe-se que a maior parte advém da iniciativa pública, podendo-se presumir que isso ocorre devido aos tipos de cursos oferecidos pelo Cerfead, em sua maior parte voltada para educação ou administração pública, ou por incentivos das instituições nas quais trabalham.

Os participantes que usam WebC atribuíram melhores notas à tecnologia usada do que os que usam a VideoC. A Figura 1 apresenta um gráfico criado a partir do índice gerado por participante. O gráfico evidencia uma média de notas superiores a 4, em uma escala de 1 a 5, em 55\% das notas atribuídas pelos participantes da WebC; para a VideoC o valor foi de $22 \%$. Foi calculada também a média de todos os índices da WebC e da VideoC, que resultou em 4,0 e 3,3, respectivamente.

Figura 1 - Percepção geral dos participantes em relação à VideoC e à WebC.

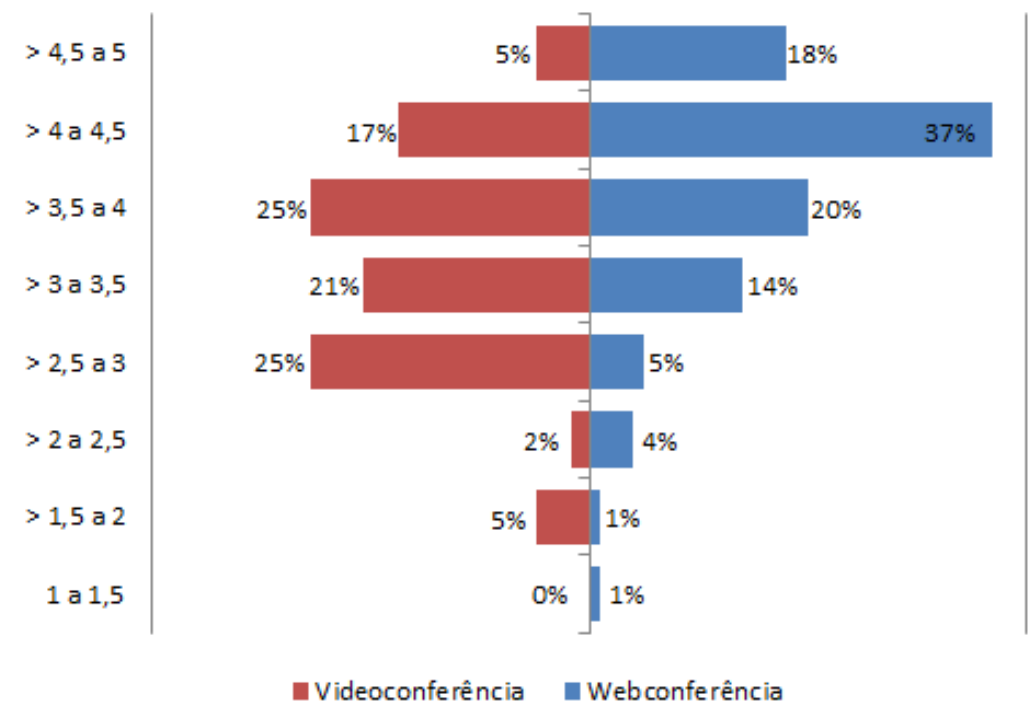

Fonte: os autores.

A Figura 2 apresenta a percepção dos participantes em relação à adequação do ambiente (Q3), influência do professor (Q4), quantidade e qualidade das interações (Q5 e Q6) e contribuição das interações síncronas na sua formação profissional. Percebe-se maior concentração de notas 4 e 5 na avaliação dos estudantes que utilizam WebC, enquanto que para a VideoC as notas concentram-se em 3 e 4 . Apenas a influência do professor obteve resultados similares para as duas tecnologias; $74 \%$ dos participantes que utilizam WebC e $78 \%$ dos que utilizam VideoC atribuíram notas 4 e 5 a essa questão. A quantidade e qualidade das interações 
nas WebC obtiveram, respectivamente, notas 4 e 5 em 69\% e 72\% dos casos; na VideoC apenas $30 \%$ e $41 \%$ dos participantes atribuíram as notas 4 e 5 nesses casos. Em relação à adequação do ambiente, o percentual de notas entre 4 e 5 da WebC é de 93\%, mais que o dobro da ocorrência na VideoC (45\%). Em relação à contribuição das conferências para a formação profissional, os resultados obtidos na WebC demonstram um nível de satisfação significativamente maior do que na VideoC. Todos esses aspectos serão mais discutidos posteriormente.

Figura 2 - Concentração de notas por questão e tecnologia de teleconferência.

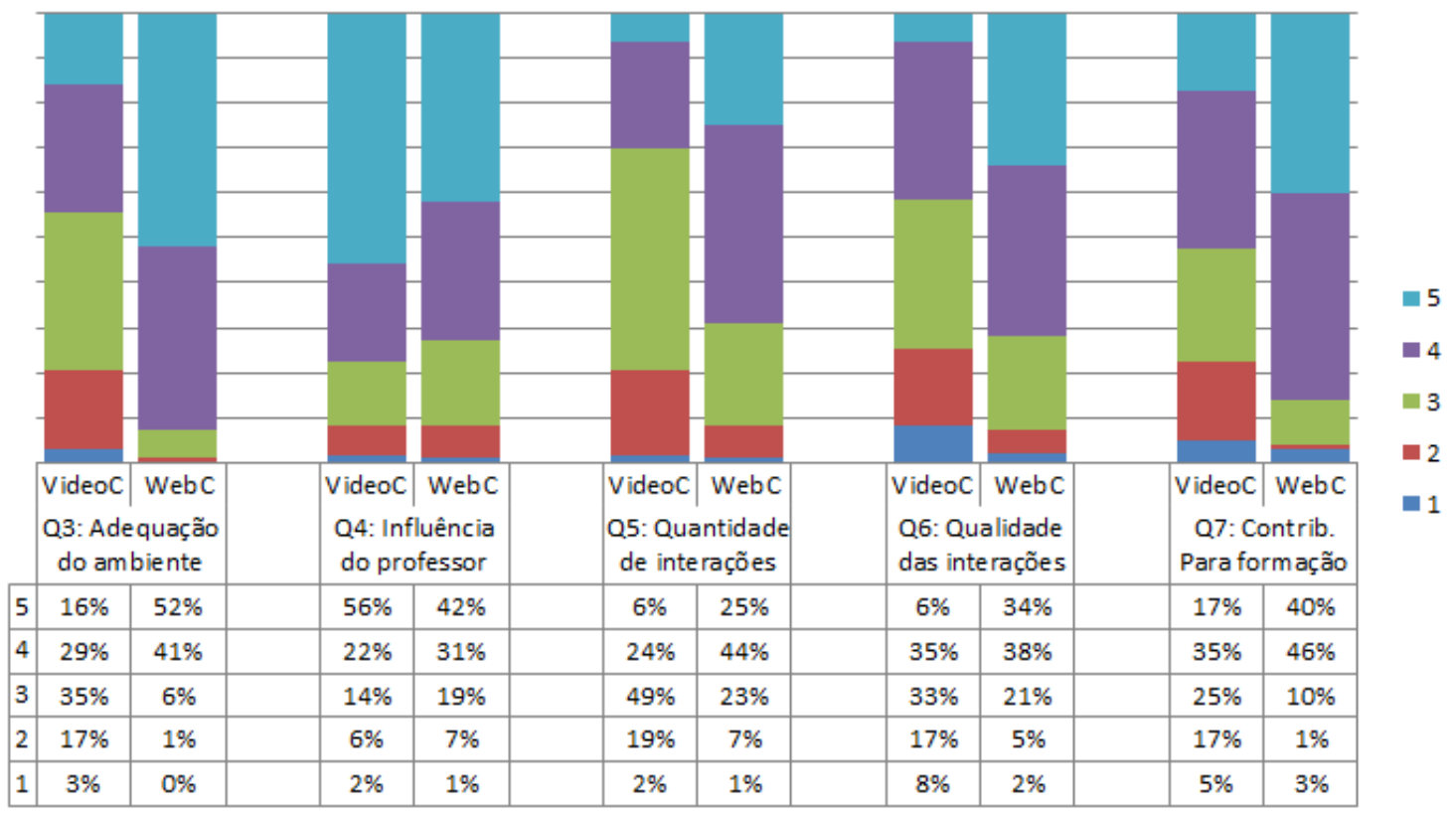

Fonte: os autores.

A Tabela 2 apresenta os indicadores estatísticos aplicados aos dados coletados, agrupados por tecnologia de teleconferência. Percebe-se certa vantagem da WebC em todos os aspectos avaliados. Comparando-se os indicadores de desvio padrão e coeficiente de variação tem-se menor valores para as WebC, o que indica que as avaliações se concentram em torno da própria média, conferindo maior uniformidade entre os participantes dessa tecnologia. Os dados da moda, que representam a nota com maior número de ocorrências na questão, são iguais ou superiores na tecnologia de WebC. Na Q3, que avalia a percepção dos estudantes em relação à adequação do ambiente ao participar da teleconferência, a moda foi 3 e 5 para VideoC e WebC, respectivamente. Este dado, conjugado com os dados apresentados na Figura 2, pode indicar deficiências nos polos de apoio presencial para receber os estudantes ou que as WebC beneficiam o conforto dos estudantes pois são eles próprios que escolhem o ambiente de estudo. Nas Q5, Q6 e Q7 também percebe-se uma diferença importante entre as duas tecnologias, corroborando o observado na Figura 2. 
Tabela 2 - Indicadores estatísticos aplicados aos dados coletados.

\begin{tabular}{|c|c|c|c|c|c|c|c|}
\hline \multirow[b]{2}{*}{$\begin{array}{l}\text { Tecnologi } \\
\text { a }\end{array}$} & \multirow[b]{2}{*}{$\begin{array}{l}\text { Indicadores } \\
\text { estatísticos }\end{array}$} & \multicolumn{6}{|c|}{ Questões } \\
\hline & & $\begin{array}{c}\text { Q2* } \\
\text { Qualidade } \\
\text { das } \\
\text { conferências }\end{array}$ & $\begin{array}{c}\text { Q3 } \\
\text { Adequação } \\
\text { do } \\
\text { ambiente }\end{array}$ & $\begin{array}{c}\text { Q4 } \\
\text { Influência } \\
\text { do } \\
\text { professor }\end{array}$ & $\begin{array}{c}\text { Q5 } \\
\text { Quantidade } \\
\text { de } \\
\text { interações }\end{array}$ & $\begin{array}{c}\text { Q6 } \\
\text { Qualidade } \\
\text { das } \\
\text { interações }\end{array}$ & $\begin{array}{c}\text { Q7 } \\
\text { Contrib. } \\
\text { para a } \\
\text { formação }\end{array}$ \\
\hline \multirow[t]{4}{*}{ VideoC } & Média & 2,7 & 3,4 & 4,2 & 3,1 & 3,1 & 3,4 \\
\hline & $\mathrm{DP} * *$ & 0,8 & 1,1 & 1,0 & 0,9 & 1,0 & 1,1 \\
\hline & Moda & 2 & 3 & 5 & 3 & 4 & 4 \\
\hline & $\mathrm{CV} * * *$ & $29 \%$ & $31 \%$ & $24 \%$ & $27 \%$ & $33 \%$ & $33 \%$ \\
\hline \multirow[t]{4}{*}{ Webc } & Média & 2,8 & 4,1 & 4,4 & 3,9 & 4,0 & 4,2 \\
\hline & $\mathrm{DP} * *$ & 0,9 & 1,0 & 0,7 & 0,9 & 1,0 & 0,9 \\
\hline & Moda & 3 & 5 & 5 & 4 & 4 & 4 \\
\hline & $\mathrm{CV} * * *$ & $31 \%$ & $24 \%$ & $16 \%$ & $24 \%$ & $24 \%$ & $21 \%$ \\
\hline
\end{tabular}

* A Q2 possui escala de 1 a 4, conforme Tabela 1, sendo 1 "Muitas vezes perdi parte das aulas por problemas técnicos" e 4 "Nunca tive problemas".

** Desvio padrão.

*** Coeficiente de variação.

Fonte: os autores.

A fim de verificar as correlações entre o perfil dos participantes e sua percepção em relação às teleconferências, bem como as possíveis correlações entre os aspectos avaliados, foram feitos alguns testes. Diferente do que era esperado, os testes mostraram que a correlação é baixa na maioria dos casos, ou seja, indica que o tempo de acesso à internet, a idade, a experiência com TICs e a quantidade de cursos EAD que fez têm baixa influência nas notas atribuídas à qualidade e quantidade de interações nas teleconferências (Q6 e Q5, respectivamente), bem como à contribuição na sua formação profissional (Q7). Ou seja, mesmo participantes com idades superiores ou com menos experiência com TICs não têm dificuldade no acesso às WebC, nas quais não há tutores para auxiliá-los. Quando se correlaciona a percepção da influência do professor na qualidade das interações (Q4), da dificuldade de acesso à teleconferência (Q1) e da qualidade de transmissão da tecnologia também não há correlação. A única questão em que houve diferença, indicando correlação média, foi a respeito da adequação do ambiente do participante quando usa a teleconferência (Q3), corroborando que há certa influência da tecnologia de teleconferência usada (Tabela 3). 
Tabela 3 - Teste de correlação entre a adequação do ambiente e a quantidade e qualidade das interações e a contribuição na formação profissional.

\begin{tabular}{lcccccc} 
& Q5 Quant. interações & Q6 Qual. interações & \multicolumn{2}{c}{ Q7 Contrib. formação } \\
Questão & VideoC & WebC & VideoC & WebC & VideoC & WebC \\
\hline $\begin{array}{l}\text { Adequação do ambiente } \\
\text { (Q3) }\end{array}$ & Média & Baixa & Média & Média & Baixa & Média \\
\hline
\end{tabular}

Fonte: os autores.

\subsection{Uso das interações síncronas}

Conforme mencionado anteriormente, as teleconferências são as principais ferramentas de interação síncrona usadas em cursos de educação a distância. Seu uso pressupõe interação com os estudantes; sem a interação, esse momento síncrono poderia ser substituído por uma vídeoaula para que os estudantes assistam a qualquer momento. Alguns estudantes do curso VL inclusive enfatizaram esse aspecto (Tabela 4).

Tabela 4 - Comentários dos estudantes do curso VL em relação às interações síncronas.

Ainda há professores unilaterais, como os que usaram e ainda usam a conferência somente para ler slides. Para isso não precisamos de conferência.

Há aulas chatas, unilaterais, sendo desnecessário o deslocamento até o polo presencial, pois é bem melhor apenas ler o conteúdo disponível no Moodle.

Outro problema que aponto aqui seria em relação ao uso de outras ferramentas que não sejam apenas slides lidos pelos professores.

Fonte: os autores.

Três questões permitiram aos participantes manifestarem mais diretamente sua percepção em relação às interações, as Q2, Q5 e Q6 (Tabela 2). As respostas à Q2, focada na qualidade de transmissão das teleconferências, demonstrou uma pequena diferença de percepção entre a VideoC e a WebC, com vantagem para esta última. No entanto, em ambos os casos a avaliação indicou a necessidade de ações para melhorar esse aspecto; mesmo na WebC, que foi melhor avaliada, a média foi de 2,8 (em uma escala de 1 a 4) e a maioria das respostas se concentrou na nota 3.

As médias obtidas pela VideoC nas Q5 e Q6, relacionadas à quantidade e qualidade das interações, também indicam a necessidade de mudanças na abordagem utilizada atualmente (Figura 2 e Tabela 2). A Tabela 3 mostra que essas duas questões estão correlacionadas à adequação do ambiente no qual os estudantes estão quando participam das VideoC, considerando o conforto, a comodidade, o nível de silêncio etc. (Q3). Sendo assim, esse pode ser um dos fatores trabalhados a fim de obter uma melhoria na percepção dos estudantes em relação aos momentos de interação síncrona. Outra possibilidade é a sugerida por Schueter, 
Bleicher e Juliani (2017) a partir de pesquisa com estudantes: intercalar momentos teóricos e práticos. Na seção 3.5, apresentam-se as sugestões feitas pelos próprios participantes do estudo e que podem orientar ações de melhoria das interações síncronas.

A considerável diferença entre a avaliação das WebC e VideoC a respeito da percepção de quantidade e qualidade das interações com o professor pode estar relacionada à atuação do professor e à tecnologia em si. Segundo Carneiro, Maraschin e Tarouco (2004), o professor precisa planejar e proporcionar os momentos de integração para propiciar a efetiva aprendizagem. Nortvig, Pettersen e Balle (2018) ressaltam que, visto que alguns estudantes atribuem menos valor às atividades online pois acham que elas demandam menos sua participação, é importante dar destaque às interações durante esses momentos. Do ponto de vista da tecnologia, enquanto na VideoC o estudante precisa levantar e se deslocar até o equipamento para falar com o professor, além de estar de frente aos estudantes do polo em que ele participa, na WebC o estudante usa o microfone do seu próprio equipamento e sua participação é totalmente online. O modo de operação da VideoC pode dificultar a participação dos estudantes mais tímidos, enquanto que nas WebC eles estão "protegidos" por acessar a conferência diretamente de seu computador.

\subsection{A importância do professor}

O professor tem forte influência na qualidade das interações síncronas com os estudantes na EAD, o que foi evidenciado pelos participantes na Q4. Nas duas tecnologias consultadas, a maioria dos participantes atribuiu notas 5 e 4 para essa questão - 73\% na WebC e 78\% na VideoC (Figura 2), sendo 5 a nota mais atribuída em ambos os casos (Tabela 2). Além disso, alguns estudantes deixaram comentários (tanto positivos quanto negativos) que corroboram essa percepção (Tabela 5).

Tabela 5 - Comentários dos participantes de sobre a importância do professor.

A interação dos professores é excelente, e sempre há muito aproveitamento nas duas horas de trabalho. (WT)

Alguns professores têm criado estratégias de, mesmo a distância, interagir com os estudantes, a que mais eu vejo como válida é a de criar situações em que os polos tenham de se posicionar ativamente durante a aula. (WT)

O conteúdo e as ferramentas do Moodle são excelentes. Os professores e a interação também. As aulas foram dinâmicas adorei a proposta. (WT)

Percebi que este contato com o professor é muito importante, pois auxilia nas dúvidas e ajuda na hora de fazer as atividades, principalmente as gravações que podem ser exibidas várias vezes. (WD)

Eu percebo em algumas situações que os professores parecem não terem testado as ferramentas antes de demonstrá-las. (WT)

Sinto falta de ter contato a mais, o único profe que permite ter um acesso com ele melhor é o prof $X$. (WT)

Sinto indigesto o professor que totaliza o slide de apresentação com texto e faz a leitura como aula. (WD) 
Fonte: os autores.

Lunkes, Selli e Prates (2008) afirmam que a configuração das interações, seja em aulas presenciais ou em ambientes virtuais, se dá de acordo com os métodos da prática docente. A atuação do professor, como um mediador do processo de aprendizagem, impacta na efetividade dos cursos EAD (BOTELHO; VICARI, 2009). Os comentários dos estudantes, bem como as notas que atribuíram à influência do professor na qualidade das interações corrobora o que Nortvig, Pettersen e Balle (2018) constataram: que uma forte presença do professor, conjugada com um conteúdo de qualidade, são elementos essenciais em cursos online que conseguem facilitar a aprendizagem e o engajamento dos estudantes. Os autores também ressaltam que o ensino em ambientes online coloca diversos desafios aos professores, os quais muitas vezes fazem um esforço para adaptar ao contexto online práticas que acham efetivas em cursos presenciais.

\subsection{Características intrínsecas aos modelos de teleconferência}

As melhores notas atribuídas pelos participantes das WebC em relação às VideoC podem estar relacionadas às características intrínsecas de cada tecnologia. No caso das VideoC, segundo Kanashiro et al. (2014), os polos de apoio presencial, principalmente os de cidades menores, carecem de melhor infraestrutura e atendimento de TI. Em relação a acesso e qualidade das VideoC, por exemplo, alguns participantes veem como desvantagem a obrigatoriedade de ir até o polo para as interações síncronas. Além disso, esperava-se que a avaliação dessa tecnologia superasse a WebC em relação à qualidade de transmissão já que são usados equipamentos específicos para esse fim, mas não foi o encontrado nos dados coletados, conforme percebe-se pelos comentários da Tabela 6 e pelas sugestões dos estudantes (Tabela 7).

Tabela 6 - Comentários sobre dificuldade de acesso e qualidade de transmissão em VideoC.

Acredito que por ser uma experiência que o acesso à internet permite em qualquer lugar a obrigatoriedade de ir até o polo para as Vídeo acaba complicando pois a maioria dos alunos tem que se deslocar de vários lugares o que não é nem sustentável, nem saudável, quando a interação pode ser realizada de lugares mais próximos ou até mesmo em sua residência. (VG)

Na sala de aula onde estamos recebendo este conteúdo, também seria possível melhorar a exibição. Hoje a imagem que aparece é muito pequena, mesmo sendo projetada por um projetor digital [...] Às vezes a imagem é tão pequena - mesmo tendo uma parede enorme para ser projetada - que nem é possível ler os conteúdos dos slides dos professores. (VL)

Os problemas técnicos da videoconferência são: em relação ao som emitido, o qual perde qualidade, e assim não se ouve, muitas vezes, de forma clara o professor. (VG)

Outro aspecto que requer um "olhar carinhoso" por parte da coordenação dos cursos EAD é o ambiente das salas de aula. Espaços inadequados para videoconferências, som com ruído, acústica péssima, conexão (internet) e questões técnicas que deixam a desejar. (VG)

Fonte: os autores.

\subsection{Contribuição para a formação profissional}


Sendo as teleconferências o momento privilegiado de interação síncrona entre professores e estudantes, e entre os próprios estudantes, e sendo os cursos estudados todos de educação profissional, foi questionado aos estudantes qual a contribuição que esses momentos têm sobre sua formação para o trabalho. Para as VideoC, $52 \%$ das notas foram 4 e 5, com a média igual a 3,4 ; já no caso das WebC, $86 \%$ das notas se concentraram nas notas 4 e 5 , e a média foi igual a 4,2, significativamente superior à observada na outra tecnologia. Aparentemente, a contribuição das interações síncronas na formação profissional dos participantes caminha junto com a quantidade e qualidade desses momentos. A integração de atividades práticas e autênticas, de formação interprofissional e de atitudes colaborativas (GRUBER et al., no prelo) podem ser maneiras de aprimorar as interações síncronas em cursos da educação profissional, aumentando o impacto que esses momentos têm sobre a formação profissional dos estudantes.

\subsection{Recomendações para melhoria das interações síncronas}

Os estudantes são capazes de identificar problemas e propor soluções consistentes (MEYEN et al., 2002). Dos 163 participantes, 20 fizeram sugestões de aprimoramento das interações síncronas, as quais foram agrupadas por tecnologia e domínio na Tabela 7.

Tabela 7 - Recomendações dos estudantes para aprimoramento das teleconferências.

\begin{tabular}{lll}
\hline & Webconferência & Videoconferência \\
\hline I & - Disponibilizar melhores equipamentos & - Aprimorar infraestrutura física e tecnológica dos \\
$\mathbf{n}$ & (por exemplo, microfones) aos & polos (acústica da sala e conexão com internet, por \\
$\mathbf{f}$ & professores. & exemplo). \\
$\mathbf{r}$ & & - Melhorar qualidade da transmissão. \\
$\mathbf{a}$ & & - Projetar a imagem da teleconferência em tamanho \\
$\mathbf{-}$ & maior. \\
$\mathbf{e}$ & \\
$\mathbf{s}$ & \\
$\mathbf{t}$ & \\
$\mathbf{r}$ & \\
$\mathbf{u}$ & \\
$\mathbf{t}$ & \\
$\mathbf{u}$ & \\
$\mathbf{r}$ & \\
$\mathbf{a}$ &
\end{tabular}


P - Testar anteriormente as ferramentas

r apresentadas em aula.

e - Enviar aos estudantes a pauta das

p teleconferências com antecedência.

a - Disponibilizar aos estudantes vídeos

r curtos explicando os objetivos principais

a da disciplina e das atividades avaliativas.

ç - Preparar apresentações de slides mais

ã interativas e instigantes.

- - Utilizar recursos mais atrativos que

d apresentações de slides durante as

a interações síncronas, como imagens,

s fluxogramas, mapas conceituais

$$
\text { dinâmicos. }
$$

n - Indicar no material didático até que

t ponto o estudante deve ler antes de uma

e determinada teleconferência.

r - Inserir legendas nos vídeos.

a - Disponibilizar gravações das aulas no ambiente virtual.

õ - Orientar os convidados externos na

e elaboração de suas apresentações.
- Falar devagar e com clareza.

M - Conduzir as aulas de forma direta e objetiva.

d - Estabelecer mais contatos com os estudantes.

- Aprimorar a forma de interação com os estudantes.
- Substituir teleconferências sem interação por videoaulas.

- Disponibilizar gravações das aulas no ambiente virtual.

- Criar chats para os estudantes de cada polo

interagirem sobre os temas das aulas.

- Promover mais visitas dos professores aos polos.

- Disponibilizar formação sobre transmissões ao vivo aos professores.

- Rever o formato da teleconferência.

- Modificar o formato das teleconferências: gravar as aulas para os estudantes assistirem posteriormente, com controle de presença online e definição de períodos para assistirem.

- Restringir os encontros presenciais ao dia de realização das avaliações.

- Organizar duas avaliações no caso de disciplinas com duração superior a 45 horas, uma intermediária e uma final.

- Usar fóruns e chats para realizar as avaliações parciais.
- Realizar interações mais intensas e com discussões baseadas em leitura prévia do material.

- Permanecer em pé e movimentar-se durante a aula. - Utilizar o tempo das interações síncronas para realização de atividades práticas, trabalhos em grupo, trocas de experiências e ideias.

- Utilizar mais os fóruns para discutir os temas e tirar dúvidas.

- Promover debates sobre o assunto nos polos, conduzidos pelos tutores. 
Fonte: os autores.

Em ambas as tecnologias, percebe-se que os estudantes apreciam a flexibilidade de ter acesso às gravações das teleconferências para estudo posterior, o que foi observado também por Crowther, Keller e Waddoups (2004). Dessa forma, eles podem estudar no seu próprio ritmo e no momento mais conveniente. Observa-se que grande parte das sugestões concentram-se na etapa de preparação, enfatizando a importância de um planejamento criterioso e contraria a ideia, infelizmente muito comum, de que a EAD é uma modalidade de menor valor e mais fácil tanto para professores, coordenadores, equipe pedagógica, quanto para os estudantes (GUEDES; MEHLECKE; COSTA, 2008). A satisfação demonstrada pelos estudantes por meio das notas atribuídas em relação às interações síncronas, bem como a qualidade das sugestões feitas por eles, evidenciam que um trabalho de qualidade está sendo feito, o qual impacta na sua formação profissional.

\subsection{Limitações dos resultados}

Pesquisas de percepção e uso de questionários qualitativos possuem limitações; o questionário, por exemplo, coleta o comportamento declarado pelos participantes e não o comportamento observado (FIGUEIREDO, 1983). Os resultados aqui apresentados foram obtidos a partir de dados amostrais, assim, estão sujeitos a certo grau de confiança. Para as WebC, cuja população considerada é de 379 estudantes, o nível de confiança é de $90 \%$ e margem de erro de $7 \%$, enquanto que para as VideoC os valores são $688,90 \%$ e $10 \%$ respectivamente. Considerou-se como população o número de estudantes regularmente matriculados e ativos nos cursos, segundo dados das coordenações do programa $U A B$ e equipe de apoio pedagógico do Cerfead.

\section{Conclusões}

Este artigo apresentou uma avaliação da percepção de estudantes de EAD em relação às interações síncronas realizadas com auxílio de teleconferência. Quando comparadas as duas ferramentas utilizadas, VideoC e WebC recebem avaliações significativamente distintas em diversos aspectos, com vantagem para a última. A influência do professor, no entanto, foi avaliada como alta em ambos os casos. Uma das diferenças fundamentais entre os cursos que utilizam WebC e aqueles que usam VideoC é a proveniência e o financiamento do professor; enquanto nos primeiros os professores são servidores efetivos do Ifsc, nos segundos eles são contratados por meio de programa de bolsas da UAB. Essa diferença, conjugada com as diferenças intrínsecas a cada uma das ferramentas de teleconferência, indicam a necessidade de diferentes abordagens de formação e orientação dos professores para prepararem e conduzirem os momentos de interação síncrona.

Percebeu-se, tanto pela quantidade de participantes quanto pela qualidade das respostas, que os estudantes dos cursos EAD estão abertos à participação em pesquisas como esta. Além de 
responderem às perguntas fechadas do questionário, 23,9\% dos participantes deixaram comentários adicionais ao final, inclusive com sugestões pertinentes para aprimoramento das interações síncronas. Estudos como este, que buscam compreender e propor melhorias, feitos por profissionais que trabalham na EAD e ouvindo os seus estudantes, ajudam a desconstruir os preconceitos com essa modalidade e demonstrar que a interação entre professores e estudantes, e entre os próprios estudantes, é fundamental no processo de formação.

\section{Agradecimentos}

Os autores agradecem aos estudantes que participaram da pesquisa, aos coordenadores dos cursos do Cerfead e da UAB, aos professores da área de Tecnologias Educacionais do Ifsc, à equipe pedagógica do Cerfead e à coordenação geral da UAB no Ifsc.

\section{Referências}

ASSOCIAÇÃO BRASILEIRA DE EDUCAÇÃO A DISTÂNCIA (ABED). Censo EAD.BR: relatório analítico da aprendizagem a distância no Brasil 2017. Curitiba: InterSaberes, 2018.

BOTELHO, Francisco Villa Ulhôa; VICARI, Rosa Maria. Evaluation of Distance Course Effectiveness: Exploring the Quality of Interactive Processes. Informática na Educação: Teoria e prática, Porto Alegre, v. 12, n. 1, p.39-46, 2009.

CARNEIRO, Mára Lúcia Fernandes; MARASCHIN, Cleci; TAROUCO, Liane Margarida Rockenbach. Os domínios de aprendizagem e o papel do professor. Revista Novas Tecnologias na Educação, [s.l.], v. 2, n. 1, p.1$10,2004$.

CASTRO, Ana Cristina de; CARULLA, Luana Martins; MELO, Ivanise. Mediação pedagógica do tutor na UAB. Projeção e Docência, [s.I.], v. 5, n. 1, p.49-57, 2014.

CROWTHER, Michael S.; KELLER, Chris C.; WADDOUPS, Gregory L.. Improving the quality and effectiveness of computer-mediated instruction through usability evaluations. British Journal of Educational Technology, [s.I.], v. 35, n. 3, p.289-303, maio 2004.

FIGUEIREDO, Nice Menezes de. Aspectos especiais de estudos de usuários. Ciência da Informação, Brasília, v. 12 , n. 2 , p.43-57, 1983.

GUEDES, Adriana Torres; MEHLECKE, Querte Teresinha Conzi; COSTA, Janete Sander. As percepções dos professores sobre o ensino a distância: uma reflexão sobre as teorias pedagógicas e a EaD. Revista Novas Tecnologias na Educação, [s.l.], v. 6, n. 2, p. 1-10, 2008.

GRUBER, Crislaine et al. Desenvolvimento de projetos de cursos na Educação Profissional: uma revisão de literatura. Boletim Técnico do Senac (no prelo).

HAAS, Célia Maria; LOPES, José Norberto Sousa. Desafios da Docência em Educação a Distância: o que dizem os professores. Informática na Educação: teoria e prática, Porto Alegre, v. 17, n. 2, p. 113-130, jul./dez. 2014.

INSTITUTO FEDERAL DE SANTA CATARINA (IFSC). Centro de Referência em Formação e EAD, 2018. Disponível em: <http://www.ifsc.edu.br/cerfead>. Acesso em: 20 nov. 2018.

INSTITUTO NACIONAL DE PESQUISAS EDUCACIONAIS ANÍSIO TEIXEIRA (INEP). Resumo técnico: Censo da Educação Superior 2015. 2. ed. Brasília: Instituto Nacional de Estudos e Pesquisas Educacionais Anísio Teixeira, 2018.

KANASHIRO, Daniela Sayuri Kawamoto et al. "Você está me ouvindo?" As condições de trabalho docente na EAD. In: SIMPÓSIO INTERNACIONAL DE EDUCAÇÃo A DISTÂNCIA, 2014, São Carlos. Anais... . São Carlos: Ufscar, 2014. p.1 - 12. 
LUNKES, Luciana; SELLI, Maribel Susane; PRATES, Camila Camargo. Interações em Ambiente Virtual de Aprendizagem. Informática na Educação: Teoria e prática, Porto Alegre, v. 11, n. 2, p.113-124, 2008.

MEYEN, Edward $L$ et al. The Online Academy formative evaluation approach to evaluating online instruction. The Internet and Higher Education, [s.I.], v. 5, n. 2, p.89-108, jun. 2002.

NORTVIG, Anne-Mette; PETERSEN, Anne Kristine; BALLE, Søren Hattesen. A literature review of the factors influencing e-learning and blended learning in relation to learning outcome, student satisfaction and engagement. The Electronic Journal of E-learning, [s.I.], v. 16, n. 1, p.46-55, 2018.

SCHUETER, Giovana; BLEICHER, Sabrina; JULIANI, Douglas Paulesky. Encontros síncronos na EAD: panorama discente sobre o uso da webconferência. In: CONGRESSO INTERNACIONAL ABED DE EDUCAÇÃO A DISTÂNCIA, 23., 2017, Florianópolis. Anais... . Florianópolis: Abed, 2017. p. 1 - 9

Recebido em dezembro de 2018.

Aprovado para publicação em setembro de 2019.

Igor Thiago Marques Mendonça

Centro de Referência em Formação e Educação a Distância (Cerfead) - Instituto Federal de Santa Catarina (IFSC), Brasil, igor@ifsc.edu.br

\section{Crislaine Gruber}

Centro de Referência em Formação e Educação a Distância (Cerfead) - Instituto Federal de Santa Catarina (IFSC), Brasil, crislaine.gruber@ifsc.edu.br 